Supporting Information

\title{
Understanding Solvent Effects on the Properties of Two-dimensional Transition Metal Dichalcogenides
}

\author{
Jungwook Choi, Hanyu Zhang, Haodong Du, and Jong Hyun Choi* \\ School of Mechanical Engineering, Purdue University \\ West Lafayette, Indiana 47907, United States \\ *E-mail: jchoi@purdue.edu
}

\section{Contents:}

1. Materials and methods

2. AFM image, height profile, and Raman spectrum of monolayer $\mathrm{MoS}_{2}$

3. PL spectra of $\mathrm{MoS}_{2}$ before and after immersion in various solvents

4. PL spectra of WSe2 before and after immersion in various solvents

5. AFM images of $\mathrm{MoS}_{2}$ and C-AFM current maps before and after immersion in solvents 


\section{Materials and methods}

\section{Preparation of Monolayer TMDCs}

$\mathrm{MoS}_{2}$ and $\mathrm{WSe}_{2}$ flakes were mechanically exfoliated from their bulk crystals (SPI Supplies and 2Dsemiconductors Inc.) onto $\mathrm{SiO}_{2} / \mathrm{Si}$ substrates for PL studies and onto ITO/glasses for C-AFM. Both substrates were cleaned by acetone and isopropyl alcohol before exfoliation followed by oxygen plasma treatment for $60 \mathrm{~s}$. After exfoliation, the samples were annealed in an argon environment at atmospheric pressure at $250^{\circ} \mathrm{C}$ for $1 \mathrm{~h}$.

\section{Immersion in Solvents}

Toluene, chloroform, and acetone were purchased from VWR. 2-propanol or isopropanol (IPA) was obtained from Fisher Science. All solvents are highly pure (over $99.5 \%$ ) and were used as received without dilution. Thus, the solvent concentrations were nearly $100 \%$. The TMDCexfoliated substrates were immersed in $\sim 2 \mathrm{~mL}$ solvents for $20 \mathrm{~min}$ and blow-dried before measurements.

\section{Optical Characterization}

PL and Raman spectra were collected with a Renishaw inVia confocal Raman microscope using a $633 \mathrm{~nm}$ HeNe laser and a 100x objective lens at room temperature. The laser spot diameter was about $1 \mu \mathrm{m}$, and a low laser power was used to avoid any damage or heating of the samples with an exposure time of $10 \mathrm{~s}$.

\section{C-AFM Characterization}

C-AFM was performed using a Bruker Dimension Icon with a PF-TUNA module and probe. To avoid localized heating by excessive currents at nanoscale junctions between the probe tip and sample, a current range was set to $\pm 24 \mathrm{nA}$ with a contact force of $\sim 20 \mathrm{nN}$ and a scan rate of 0.5 Hz. The ITO has sheet resistance of 70-100 $\Omega /$ sq which has a thickness of about $15-30 \mathrm{~nm}$. 


\section{AFM image, height profile, and Raman spectrum of monolayer $\mathrm{MoS}_{2}$}
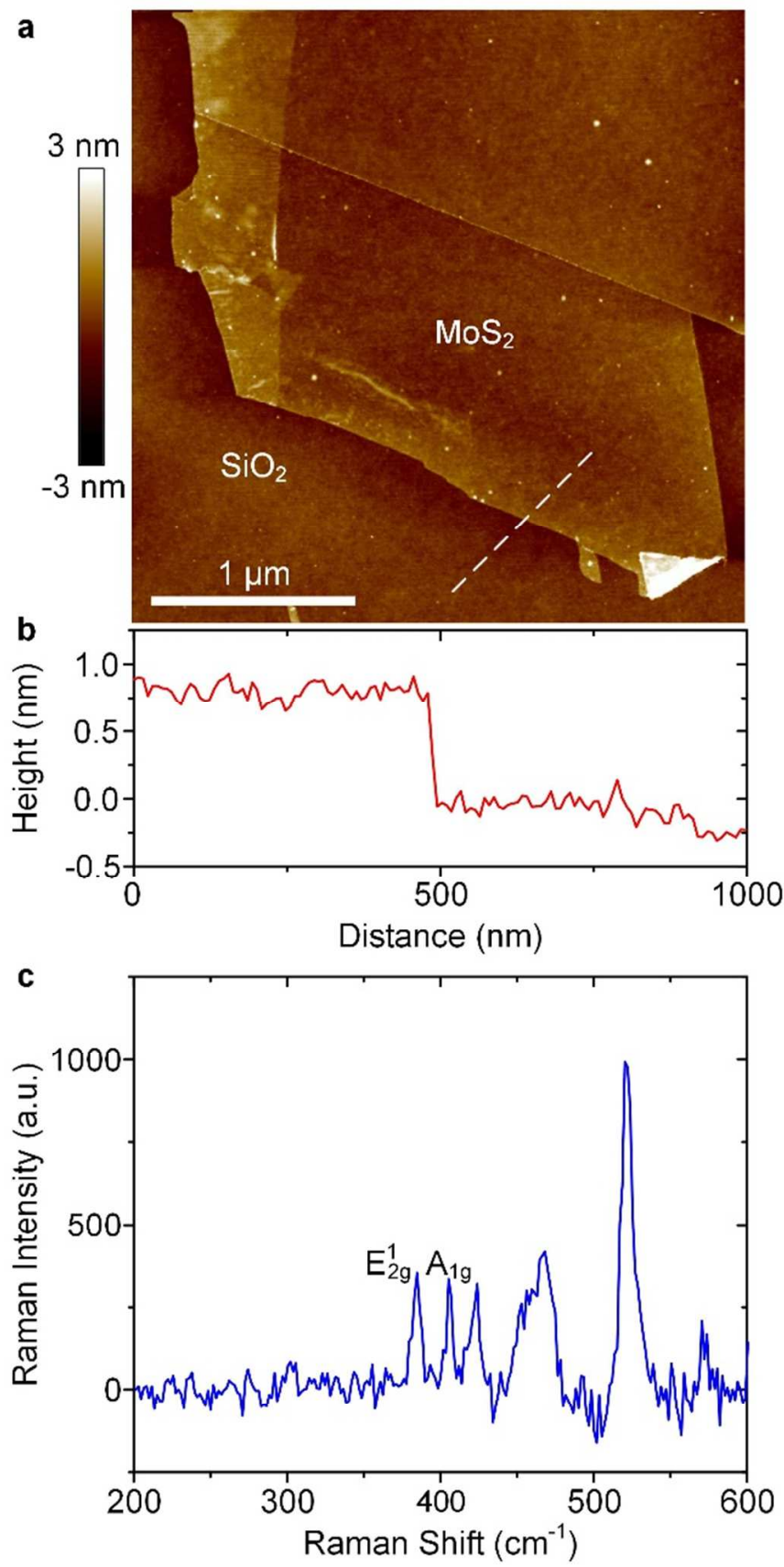

Figure S1. (a) AFM image of monolayer $\mathrm{MoS}_{2}$ exfoliated on a $\mathrm{SiO}_{2} / \mathrm{Si}$ substrate. (b) Height profile measured along the white dotted line in (a). Measured thickness of $\sim 0.8 \mathrm{~nm}$ indicates the monolayer $\mathrm{MoS}_{2}$. (c) Raman spectrum of the $\mathrm{MoS}_{2}$ monolayer excited at $633 \mathrm{~nm}$ in the ambient environment. Several Raman signatures are observed including first-order in-plane mode $\left(E_{2 \mathrm{~g}}^{1}\right)$ at around $384.8 \mathrm{~cm}^{-1}$ and out-of-plane mode $\left(\mathrm{A}_{1 \mathrm{~g}}\right)$ at $\sim 405.2 \mathrm{~cm}^{-1}$. 


\section{PL spectra of $\mathrm{MoS}_{2}$ before and after immersion in various solvents}
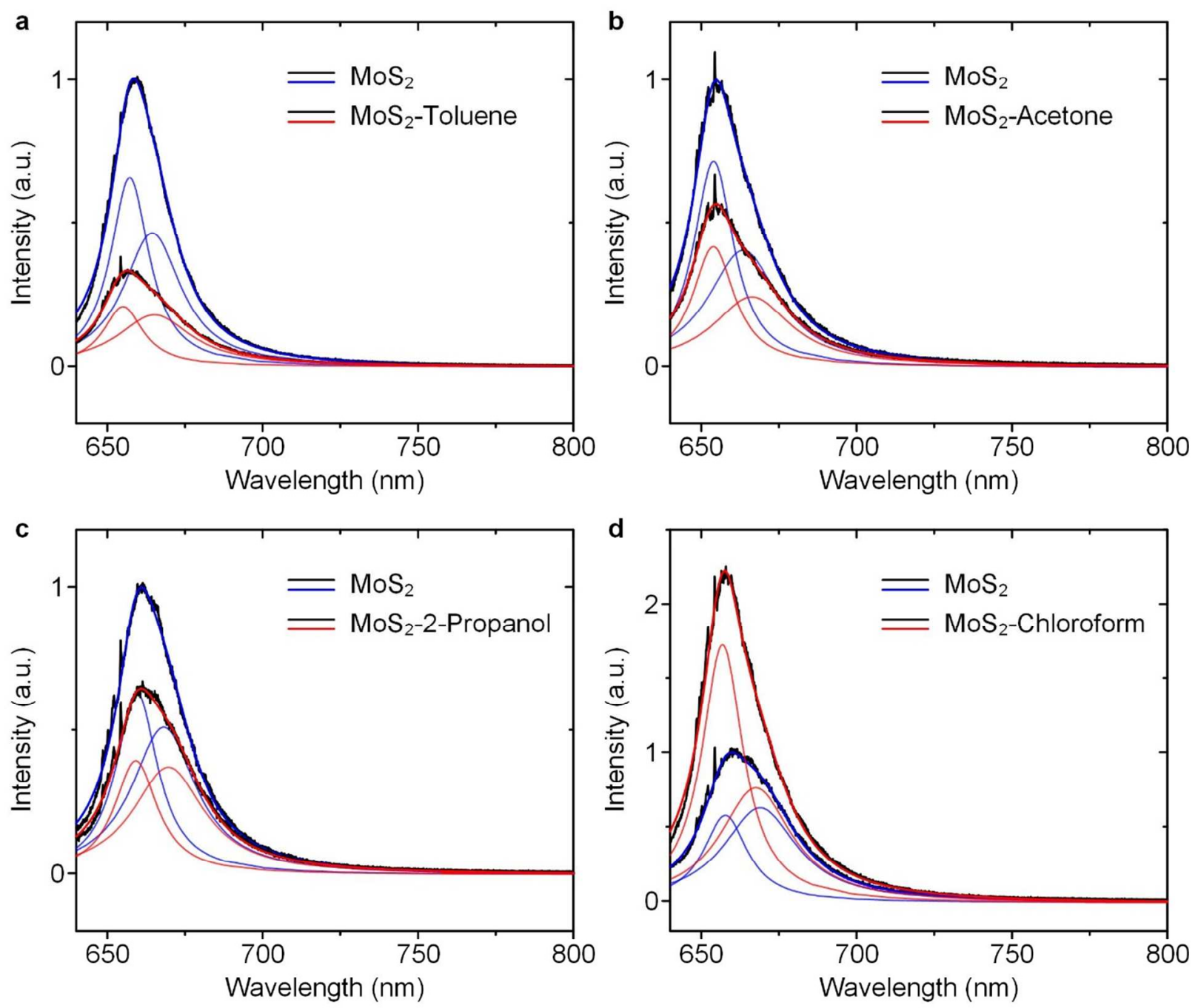

Figure S2. Representative PL spectra of $\mathrm{MoS}_{2}$ before and after immersion in (a) toluene, (b) acetone, (c) 2-propanol, and (d) chloroform. The emission measurements were performed with $633 \mathrm{~nm}$ HeNe laser excitation in the ambient environment. All spectra are normalized to the PL intensity of pristine $\mathrm{MoS}_{2}$ and deconvoluted into two Lorentzian emission peaks of exciton (X $656 \mathrm{~nm})$ and negative trion $\left(\mathrm{X}^{-} \sim 669 \mathrm{~nm}\right)$. Overall $\mathrm{PL}$ intensity decreases when $\mathrm{MoS}_{2}$ is immersed in toluene (a), acetone (b), and 2-propanol (c), whereas chloroform-immersed $\mathrm{MoS}_{2}$ (d) exhibits an enhanced PL. Toluene, acetone, and 2-propanol donate electrons to $\mathrm{MoS}_{2}$, thereby increasing trion spectra weight. In contrast, the electron withdrawing ability of chloroform suppresses formation of trion and enhances exciton recombination. 

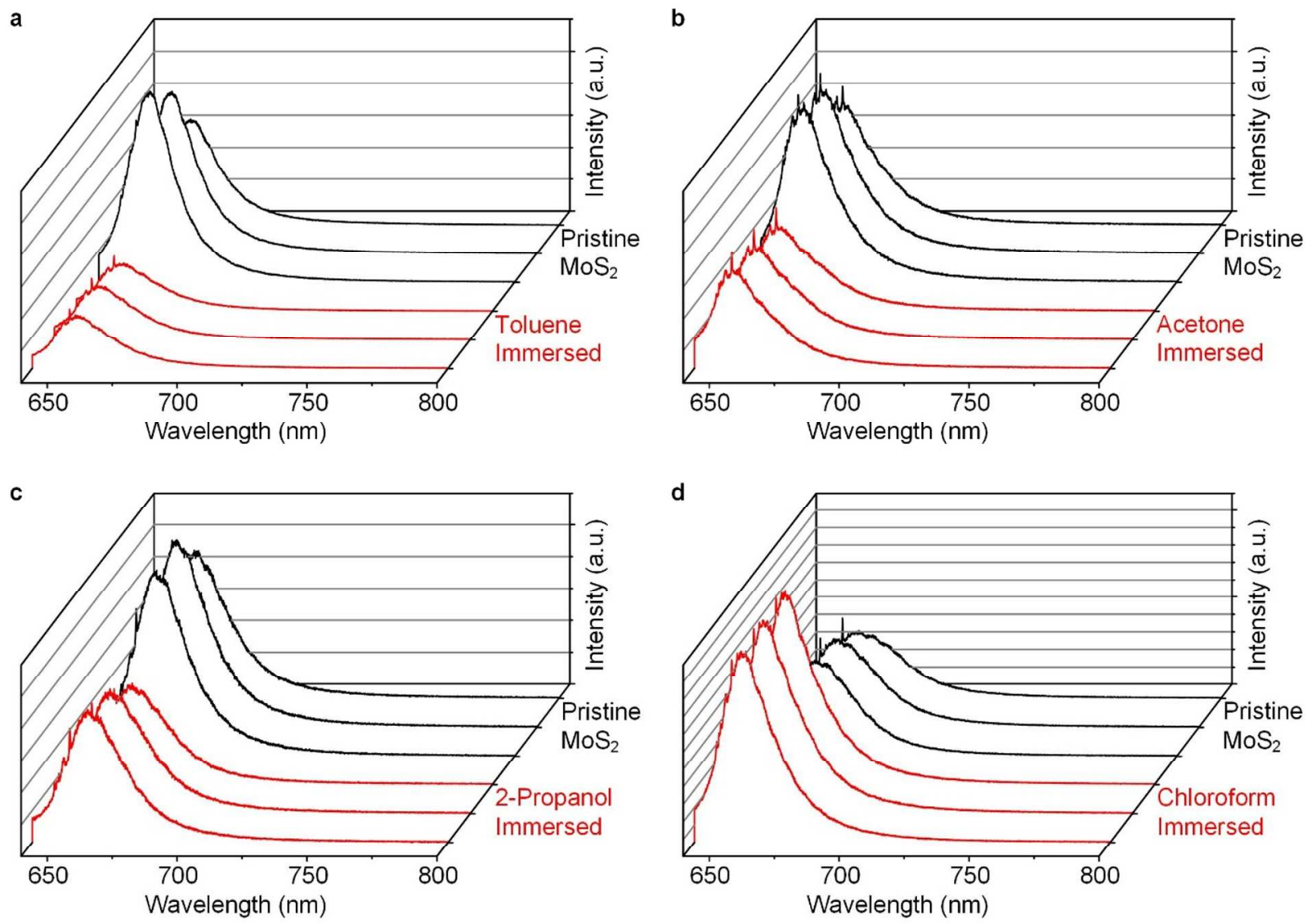

Figure S3. The PL spectra of $\mathrm{MoS}_{2}$ measured before and after immersion in (a) toluene, (b) acetone, (c) 2-propanol, and (d) chloroform. For each solvent, the PL spectra were recorded 3 times from the flake before and after immersion. The maximum variations in the PL intensity of pristine and solvent-immersed $\mathrm{MoS}_{2}$ are about 35 and 6\% (a), 32 and 10\% (b), 25 and 20\% (c), and 33 and $3 \%$ (d) for toluene, acetone, 2-propanol, and chloroform, respectively. The standard deviations of these variations are reflected as the error bars in Figure $2 \mathrm{~d}$ and e. Despite the measurement uncertainties, the changes of PL intensity after solvent immersion are clearly observable. 
a

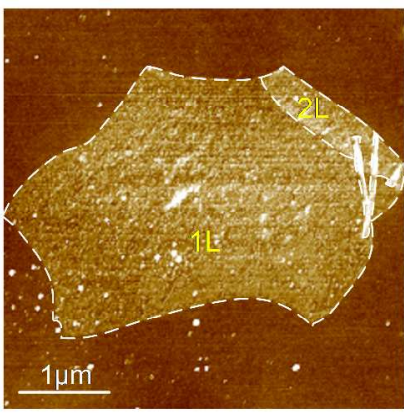

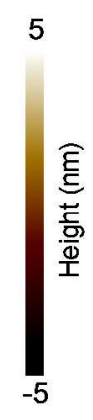
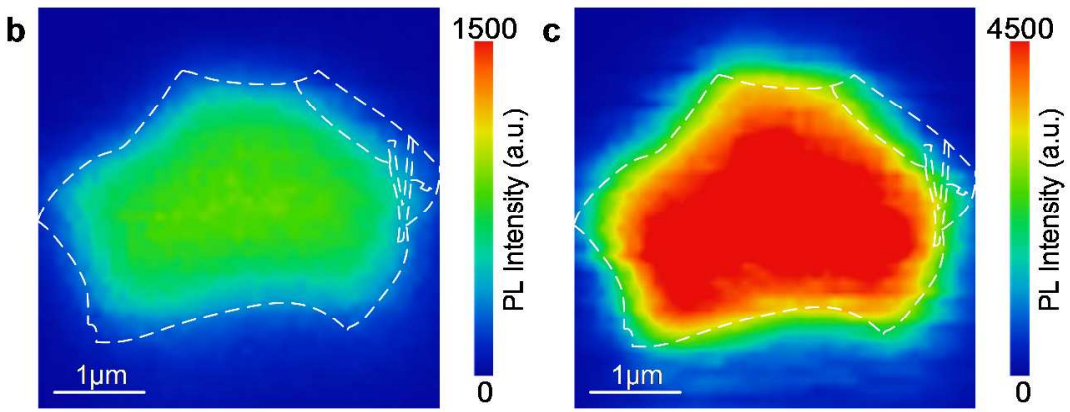

Figure S4. Spatially resolve PL measurements. (a) AFM height image of $\mathrm{MoS}_{2}$ and corresponding 2D PL intensity maps (b) before and (c) after immersion in chloroform. These maps were reconstructed based on the emission at $662 \mathrm{~nm}$. The PL intensity change after chloroform immersion is much more significant than the variation of PL intensity across the $\mathrm{MoS}_{2}$ flake. 

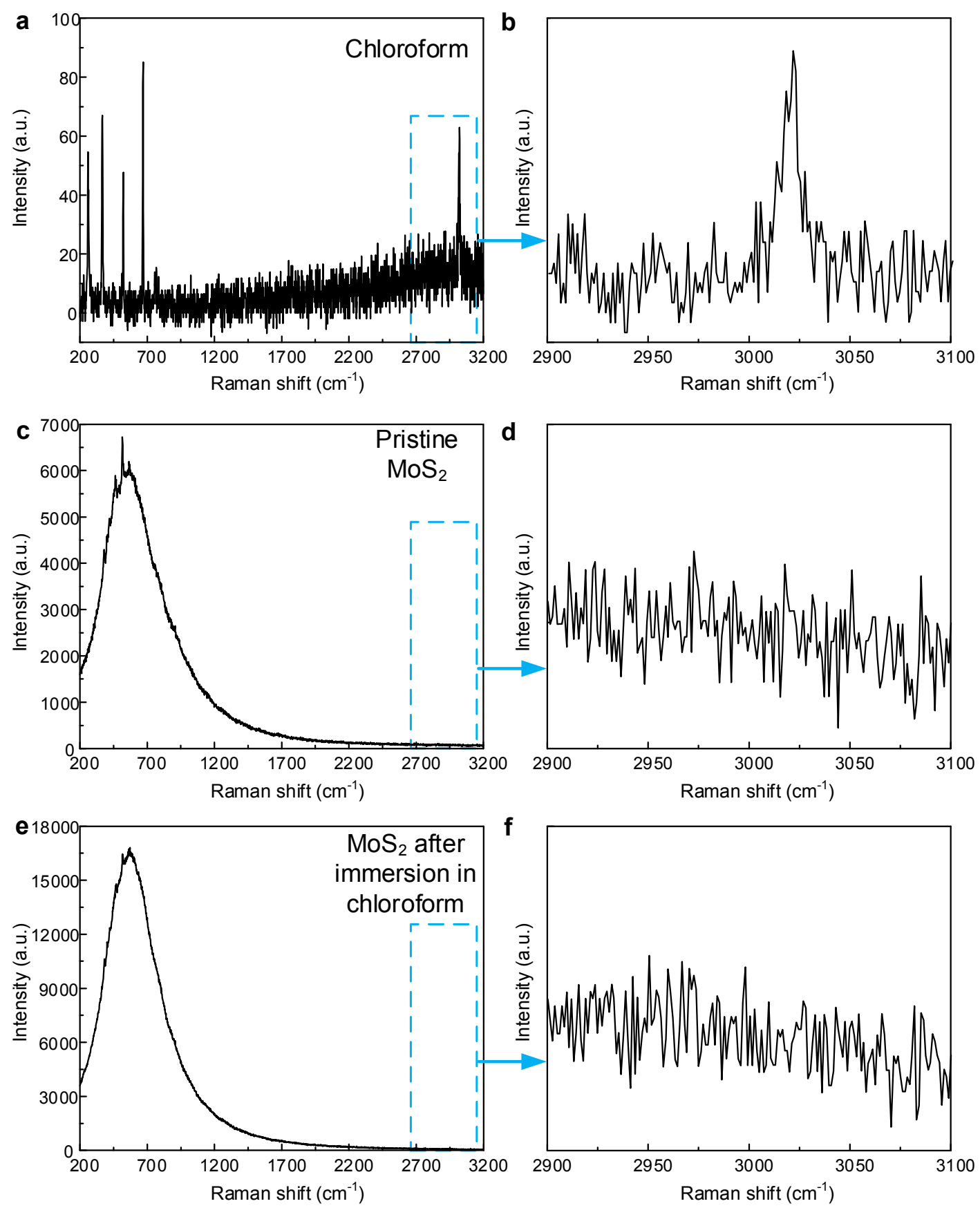

Figure S5. Raman spectra of liquid chloroform (a,b), pristine $\mathrm{MoS}_{2}(\mathrm{c}, \mathrm{d})$, and $\mathrm{MoS}_{2}$ after chloroform immersion and blow-drying (e,f). The prominent Raman signature of chloroform is observed at $\sim 3020 \mathrm{~cm}^{-1}$ [Chem. Eur. J. 2010, 16, 9010-9017]. However, this peak is completely absent in the Raman spectra of $\mathrm{MoS}_{2}$ before and after immersion in chloroform, indicating that there are no detectable solvent residues on the $\mathrm{MoS}_{2}$ flake after immersion and blow-drying. 


\section{PL spectra of $\mathrm{WSe}_{2}$ before and after immersion in various solvents}
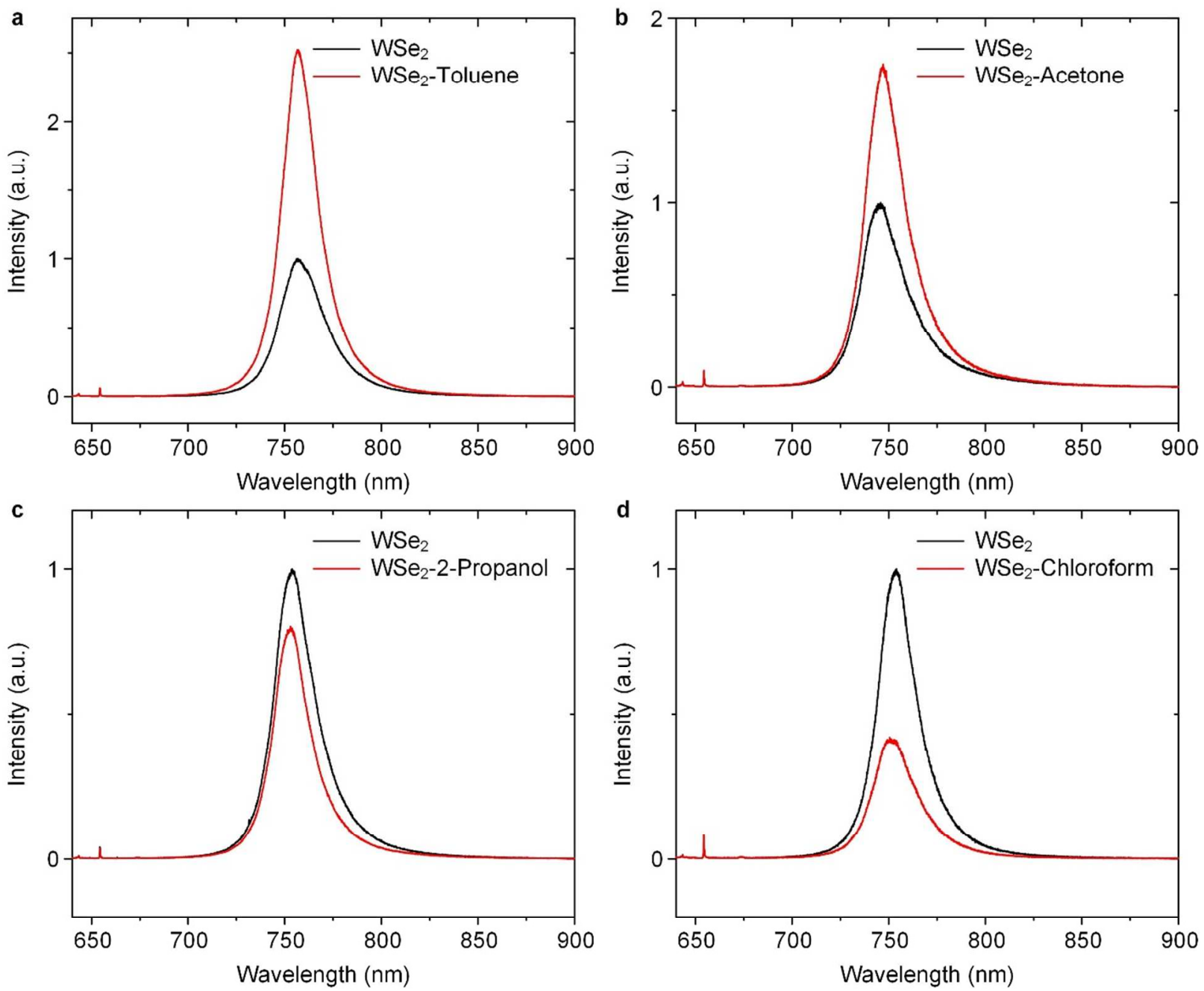

Figure S6. Representative PL spectra of $\mathrm{WSe}_{2}$ before and after immersion in (a) toluene, (b) acetone, (c) 2-propanol, and (d) chloroform. The emission measurements were performed with $633 \mathrm{~nm} \mathrm{HeNe}$ laser excitation in the ambient environment. All spectra are normalized to the PL intensity of pristine $\mathrm{WSe}_{2}$. Unlike n-type $\mathrm{MoS}_{2}$, the PL intensity of p-type $\mathrm{WSe}_{2}$ increases with electron-donating solvents such as toluene (a) and acetone (b) due to a decreased number of holes and recombination of positive trion. In contrast, holes are accumulated (i.e. electrons are depleted) by 2-propanol (c) and chloroform (d), increasing positive trion and quenching $\mathrm{WSe}_{2}$ PL. 

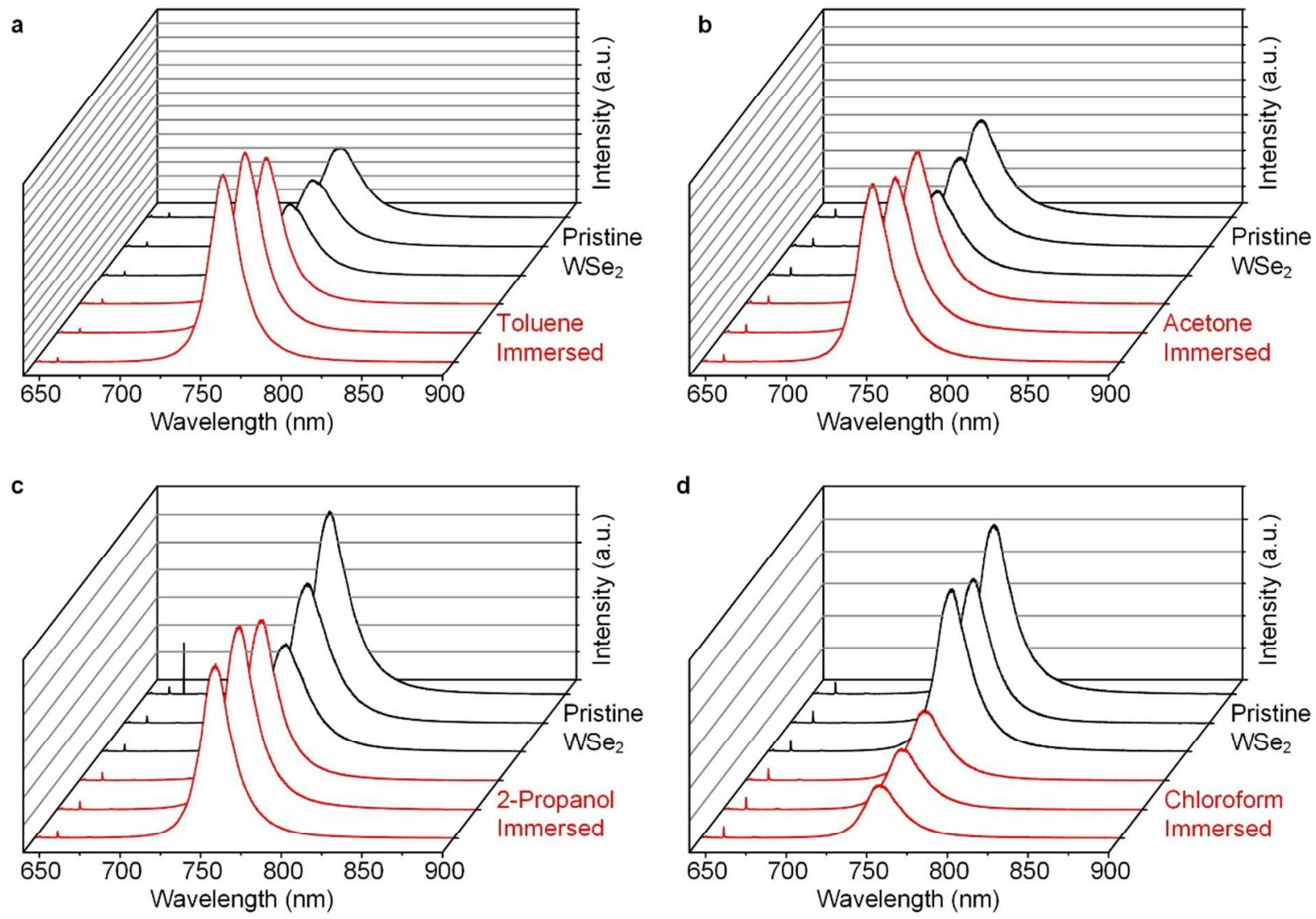

Figure S7. The PL spectra of $\mathrm{WSe}_{2}$ measured before and after immersion in (a) toluene, (b) acetone, (c) 2-propanol, and (d) chloroform. The maximum variations in PL intensity of pristine and solvent-immersed $\mathrm{WSe}_{2}$ are about 9 and 29\% (a), 15 and 27\% (b), 43 and 28\% (c), and 19 and $9 \%$ (d) for toluene, acetone, 2-propanol, and chloroform, respectively. From these variations, the standard deviations are determined as shown in the error bars in Figure 3. 


\section{AFM images of $\mathrm{MoS}_{2}$ and C-AFM current maps before and after immersion in solvents}
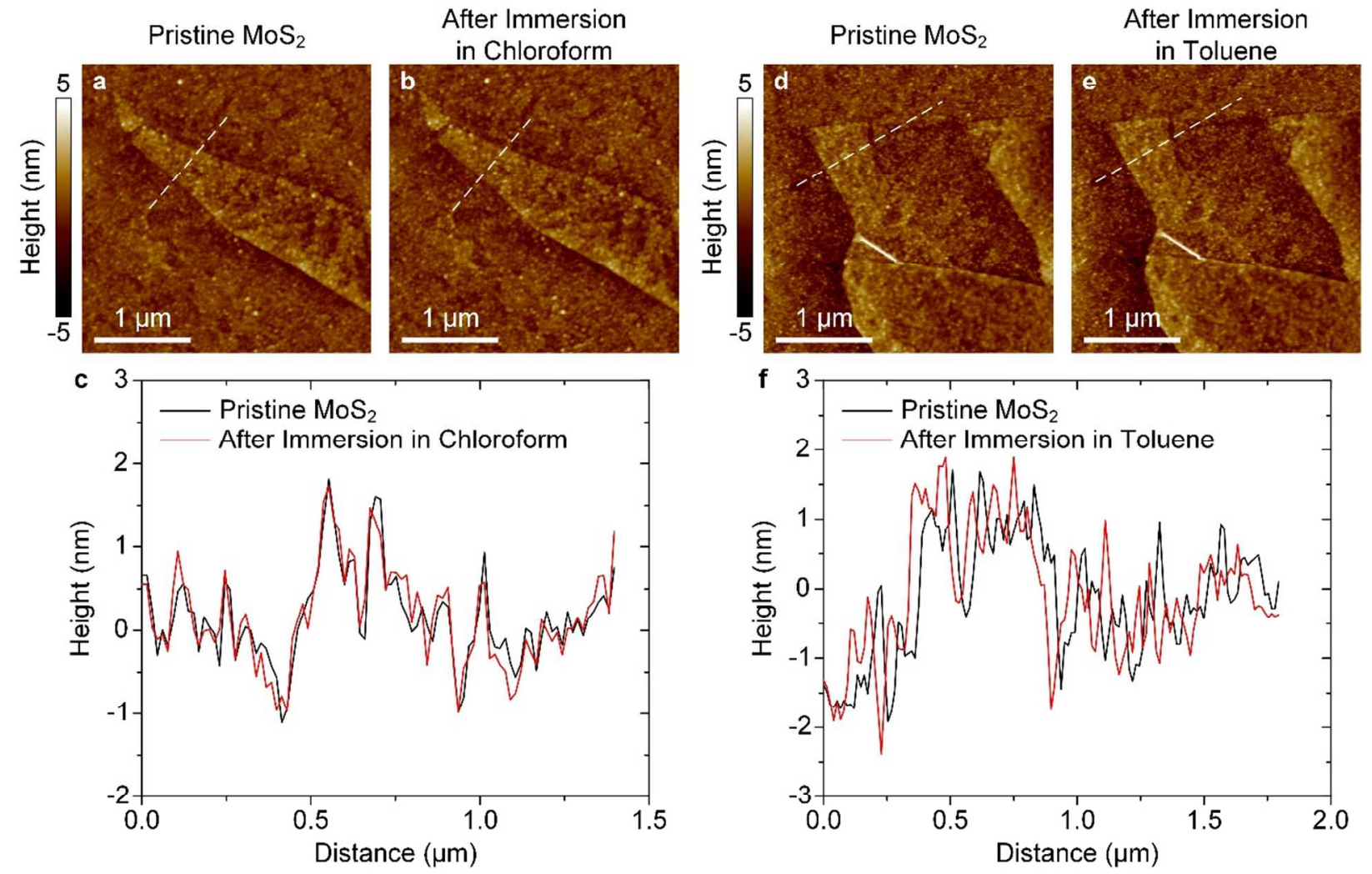

Figure S8. AFM images and height profiles of $\mathrm{MoS}_{2}$ on the ITO substrate before and after immersion in solvents. AFM image of $\mathrm{MoS}_{2}$ (a) before and (b) after immersion in chloroform, and (c) corresponding height profiles. AFM images of $\mathrm{MoS}_{2}$ (d) before and (e) after immersion in toluene, and (f) corresponding height profiles. The height profiles in (c) and (f) are measured along the white dotted lines in $(a, b)$ and $(d, e)$. There are no observable changes in height profiles of $\mathrm{MoS}_{2}$ after solvent immersion and blow-drying. 

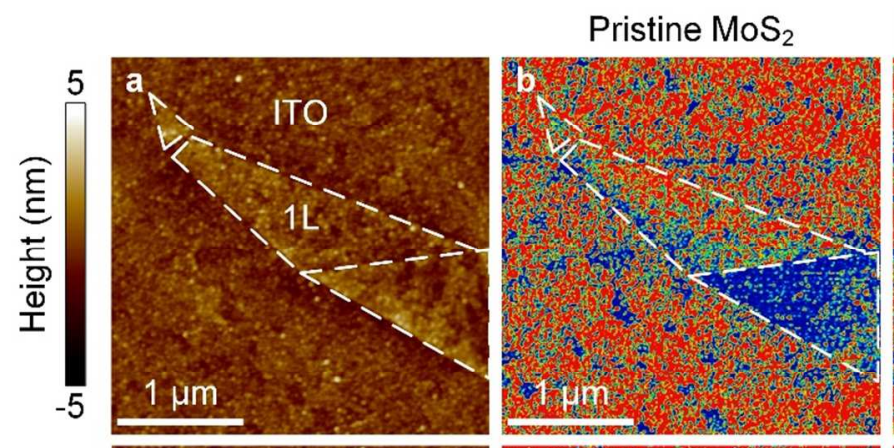

Solvent Immersed $\mathrm{MoS}_{2}$
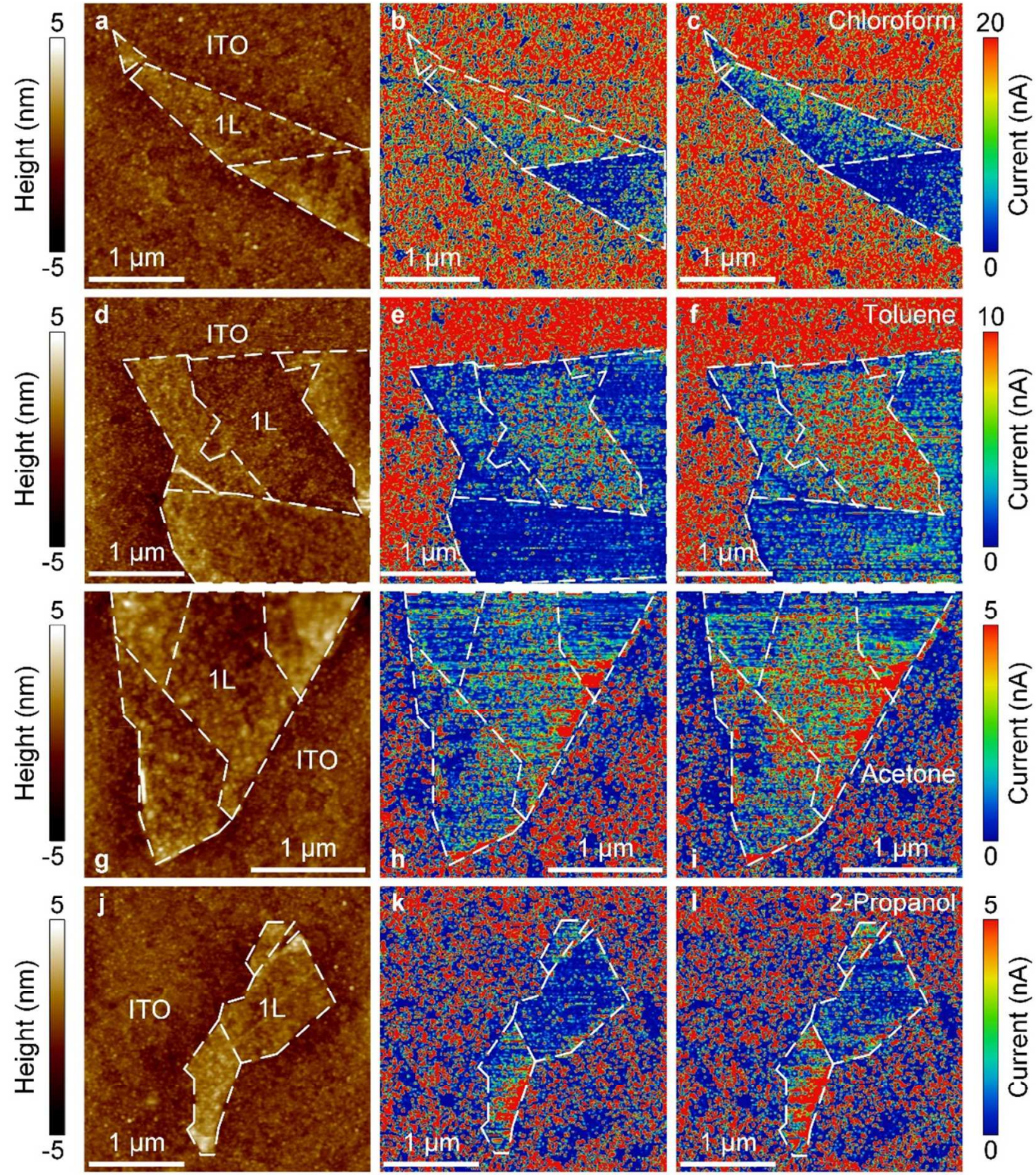

Figure S9. AFM height images and corresponding C-AFM current maps of monolayer $\mathrm{MoS}_{2}$ measured before and after immersion in $(\mathrm{a}-\mathrm{c})$ chloroform, $(\mathrm{d}-\mathrm{f})$ toluene, $(\mathrm{g}-\mathrm{i})$ acetone, and $(\mathrm{j}-\mathrm{l})$ 2-propanol. The current difference maps in Figure $4 \mathrm{~b}$ and $4 \mathrm{~d}$ of the main text are reconstructed by subtracting the pristine current map from the solvent-immersed current map at an identical bias voltage of $1.5 \mathrm{~V}$ for $(\mathrm{a}-\mathrm{c})$ and $0.5 \mathrm{~V}$ for $(\mathrm{d}-1)$. As verified by PL study, the electron density in $\mathrm{MoS}_{2}$ significantly decreases after interacting with chloroform, and thus, the current flow is also decreased as visualized in (b) and (c). Electron donating solvents such as toluene, acetone and 2-propanol increase current flow in $\mathrm{MoS}_{2}$ given the difference in electronegativity as shown in $(\mathrm{e}, \mathrm{f}),(\mathrm{h}, \mathrm{i})$, and $(\mathrm{k}, \mathrm{l})$. Relative changes of current in $\mathrm{MoS}_{2}\left(\Delta i / i_{0}\right)$ are $-0.5 \pm 0.28,0.88 \pm 0.28$, $0.56 \pm 0.51$, and $0.29 \pm 0.71$ for chloroform, toluene, acetone, and 2-propanol, respectively. 
The exfoliated $\mathrm{MoS}_{2}$ is adhered on the ITO surface via van der Waals interaction. This van der Waals force should be high enough to maintain the location of $\mathrm{MoS}_{2}$ as previously verified in mechanical deformation experiments where the suspended $\mathrm{MoS}_{2}$ was deformed by an AFM probe without sliding [ACS Nano 2011, 5, 9703-9709]. In addition, the C-AFM is usually adopted for investigating electrical defects on the surface by visualizing spatial current information. We used the thin ITO which has about $15-30 \mathrm{~nm}$ in thickness, and it could have an in-plane variation of conductivity depending on the fabrication conditions. We observed these non-uniform electrical characteristics (i.e., conductivity) of our ITO surface; defective parts showed relatively small currents than other areas as seen in C-AFM images of Figure S9. By referencing those low current areas on the ITO surface, we can identify relative locations of our $\mathrm{MoS}_{2}$ flakes, which confirms that the $\mathrm{MoS}_{2}$ flakes do not move or slide during the C-AFM measurements. Most importantly, even though there are sample-to-sample variations on the ITO conductivity, we can investigate the solvent effects on electrical properties of TMDCs, and the solvent-induced current changes are far more significant than the variations in background currents on ITO.

Changes in adhesion between the $\mathrm{MoS}_{2}$ flake and the ITO would lead to changes in contact resistance and resulting current values at identical bias voltages. In general, thermal annealing improves adhesion and contact quality at the electrical junctions. We annealed all our samples before measurements. However, the immersion in solvents may not improve the adhesion to the substrate surfaces. Given the usual distance in van der Waals interaction of $\sim 0.5 \mathrm{~nm}$, the permeation of solvents at the TMDC-substrate junction may not be allowed. In parallel, we observed opposite behaviors of current changes in $\mathrm{MoS}_{2}$ after immersion in solvents. For example, chloroform immersion depletes electrons and results in decreased currents in $\mathrm{MoS}_{2}$, while toluene donates electrons and increases current flows under identical experimental conditions. Our observation proves that solvents can modulate the electrical properties of $\mathrm{MoS}_{2}$ rather than change contact quality. 

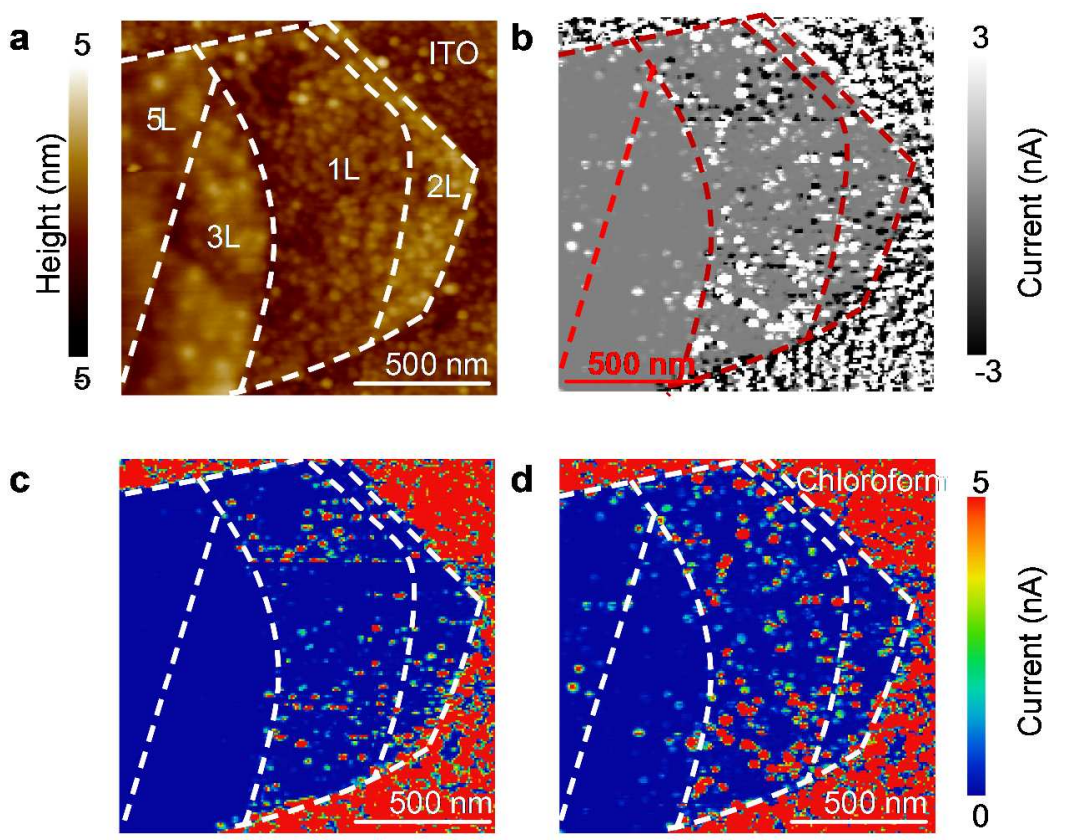

Figure S10. AFM height image of $\mathrm{WSe}_{2}$ (a), and corresponding current difference map (b) reconstructed by subtracting the current of pristine $\mathrm{WSe}_{2}$ (c) from the current map of chloroformimmersed $\mathrm{WSe}_{2}$ (d). Monolayer $\mathrm{WSe}_{2}$ shows an increase of currents after immersion in chloroform. 

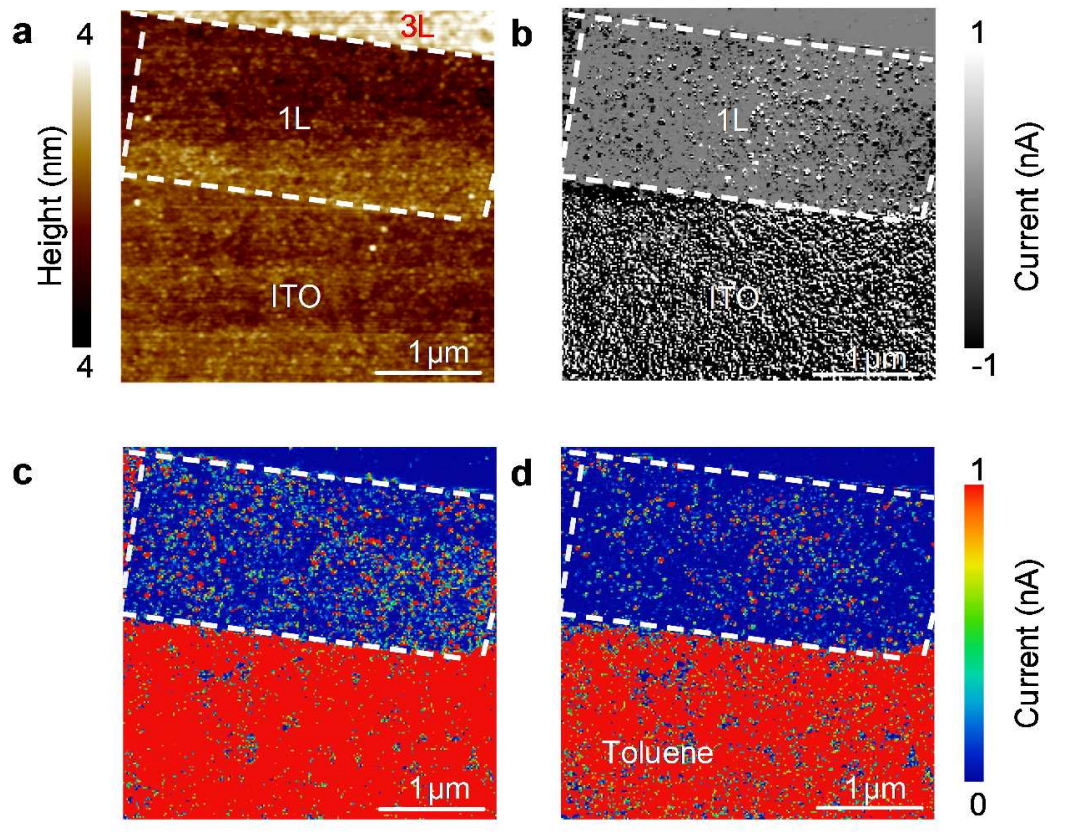

Figure S11. AFM height image of $\mathrm{WSe}_{2}$ (a), and corresponding current difference map (b) reconstructed by subtracting the current of pristine $\mathrm{WSe}_{2}$ (c) from the current map of chloroformimmersed $\mathrm{WSe}_{2}$ after immersion in toluene (d). Monolayer $\mathrm{WSe}_{2}$ shows decreased currents after immersion in toluene. 